\title{
Varieties of soluble groups
}

\section{J.R.J. Groves}

P. Hall has described certain properties of soluble groups by means of dichotomies of commutator-subgroup functions. Call a variety defined by a commutator-subgroup function a Hall variety. The aim of the thesis is to prove results concerning soluble varieties of groups which give extensions of dichotomies of $\mathrm{Hall}$ varieties.

In the first half of the thesis, we are interested in the dichotory which splits Hall varieties into those which are nilpotent and those which contain the variety of all metabelian groups. In Chapter 3 , for example, one of the results shown is that a soluble variety is nilpotent by finite exponent precisely if it does not contain, for any prime $p$, the variety of all extensions of elementary abelian p-groups by abelian groups. (This, of course, gives a dichotomy distinguishing the property 'locally max'.) In Chapter 4, the following problem is approached: is it true that a soluble variety is finite exponent by nilpotent by finite exponent if and only if it does not contain the variety of all metabelian groups? An affirmative answer is given if either the variety is metanilpotent or all the abelian by nilpotent of class 2 by abelian subvarieties of the variety can be generated by the finite groups they contain (and it appears that no variety is known which does not satisfy the latter condition).

In the second half of the thesis, we are concerned with the dichotomy which splits Hall varieties into those which are abelian by nilpotent and those which contain the variety of all centre by metabelian groups. Let $X$ denote the free group of rank 2 and, for each prime $p$, let $N_{p}$ denote the normal subgroup of $X$ generated by $\left[X^{\prime \prime}, X\right]$ and the $p$-th powers of elements of $X^{\prime}$ (or 4-th powers if $p=2$ ). Denote by $\frac{c}{p}$ the variety

Received $14 \mathrm{Julv}$ 1971. Thesis submitted to the Australian National University, May 1971. Degree approved, August 1971. Supervisors: Dr L.G. Kovács, Dr R.A. Bryce. 
generated by $X / N_{p}$. Then, using the crown product construction of B.H. Neumann, it is shown in Chapter 5 that a centre extended by abelian by nilpotent variety is abelian by nilpotent by finite exponent precisely if it contains no $\mathrm{C}_{p}$. Chapter 6 is concerned with extending this result to metanilpotent varieties. In fact the bulk of the extension is achieved, for it is shown that the finitely generated groups of a metanilpotent variety have $\max -n$ (alternatively, are residually finite) precisely if the variety contains no $\frac{c}{p}$.

The contents of Chapter 4 appear in [1] and the contents of Chapter 3 in [2].

\section{References}

[1] J.R.J. Groves, "On varieties of soluble groups", Bull. Austral. Math. Soc. 5 (1971), 95-109.

[2] J.R.J. Groves, "Varieties of soluble groups and a dichotomy of $P$. Hall", Bull. Austral. Math. Soc. 5 (1971), 391-410. 